\title{
Risk factors for acute kidney injury after unilateral radical nephrectomy and inferior vena cava thrombectomy under cardiopulmonary bypass: a retrospective study
}

Qi Zhang

Jilin Cancer Hospital

Ming Li

Jilin Cancer Hospital

Dongmei Fu

Jilin Cancer Hospital

Dongxin Wang

Jilin Cancer Hospital

Shiqi Diao ( $\nabla$ dsq66668888@163.com )

Jilin Cancer Hospital

\section{Research Article}

Keywords: renal cancer, cardiopulmonary bypass, thrombectomy, acute kidney injury

Posted Date: April 5th, 2021

DOI: https://doi.org/10.21203/rs.3.rs-381926/v1

License: (c) (i) This work is licensed under a Creative Commons Attribution 4.0 International License.

Read Full License 


\section{Abstract}

In patients with renal cell carcinoma (RCC) and cancer-related thrombosis in the inferior vena cava (IVC) or right atrium (AT), it is still unknown whether nephrectomy, anesthesia, and surgical trauma can cause postoperative acute kidney injury (AKI) and what are the risk factors for AKI. To examine the incidence and risk factors of postoperative AKI in patients who underwent unilateral radical nephrectomy and cardiopulmonary bypass (CPB)-assisted thrombectomy in the IVC and/or atrial AT due to RCC complicated with cancer-associated thrombosis. This retrospective study included patients who underwent unilateral radical nephrectomy and CPB-assisted thrombectomy in the inferior vena cava and/or atrial pulmonary artery due to RCC, under general anesthesia, from December 2011 to June 2015, at Peking University First Hospital. Among 31 patients, 15 (48.4\%) had postoperative AKI. Compared with the non-AKI group $(n=16)$, patients in the AKI group $(n=15)$ were older $(59.0 \pm 8.7$ vs. $48.5 \pm 12.9$ years, $P$ $=0.012)$ had smaller intraoperative urine volume $(1225 \pm 639$ vs. $1685 \pm 597 \mathrm{~mL}, \mathrm{P}=0.048)$. There were no differences in preoperative creatinine clearance. Age $(\mathrm{OR}=1.10,95 \% \mathrm{Cl}: 1.02-1.20, \mathrm{P}=0.020)$ was independently associated with AKI occurrence. The patients undergoing unilateral radical nephrectomy and CPB-assisted IVC thrombectomy have a high rate of AKI. Older ones are at a higher risk of postoperative AKI.

\section{Introduction}

Renal cell carcinoma (RCC) is a heterogeneous group of kidney cancers comprising many histologic subtypes, with clear cell histology being the most common subtype ${ }^{1}$. Of all cancers in the urinary system, RCC accounts for about $3 \%$ of all cancers ${ }^{2}$, with estimated 403,262 new cases and 175,098 deaths in $2018^{3}$. RCC most commonly affects elderly persons aged $60-70$ years ${ }^{1,4}$. Men are twice as likely to be affected as women ${ }^{3}$. The cause of the disease is likely multifactorial, involving genetic predisposition and environmental influences. The most common risk factors include tobacco smoking, hypertension, obesity, and genetic risk factors such as von Hippel-Lindau disease 1,4,5. Most renal cell carcinomas are detected incidentally. For nonincidental detection, typical presentation is a triad of flank pain, gross hematuria, and palpable abdominal mass ${ }^{1,4,5}$. The 5 -year relative survival of patients with kidney and renal pelvic cancer is $93 \%$ for localized disease, $69 \%$ for regional disease, and $12 \%$ for the distant-stage disease ${ }^{6}$.

RCC complicated with cancer-associated thrombosis in the renal vein or inferior vena cava or even in the right atrium is a challenge in RCC treatment ${ }^{7-10}$. Vascular invasion occurs in about $10 \%$ of the cases of $\mathrm{RCC}^{9}$ and drastically change the staging (stage 3 , irrespective of tumor size), prognosis, and surgical strategy ${ }^{9}$. The surgical strategy for such RCCs is radical nephrectomy combined with inferior vena cava or right atrial thrombectomy, which, in difficult cases, is performed with the help of cardiopulmonary bypass (CPB) ${ }^{8,11}$. 
It is still unknown in such patients whether nephrectomy, anesthesia, and surgical trauma can cause postoperative acute kidney injury (AKI) and the risk factors for $\mathrm{AKI}^{12}$. Therefore, this study aimed to examine the incidence and risk factors of postoperative AKI in patients who underwent unilateral radical nephrectomy and CPB-assisted thrombectomy in the inferior vena cava and/or atrial pulmonary artery due to RCC complicated with cancer-associated thrombosis in the inferior vena cava or right atrium.

\section{Results}

\section{Characteristics of the patients}

This study included 31 patients (17 males and 14 females). Fifteen (48.4\%) had postoperative AKI (Table 1). Compared with the non-AKI group, patients in the AKI group were older ( $59.0 \pm 8.7$ vs. $48.5 \pm 12.9$ years, $\mathrm{P}=0.012)$. There were no differences in $\mathrm{Ccr}(\mathrm{P}=0.705)$. Compared with the non-AKI group, patients in the AKI group had smaller intraoperative urine volume ( $1225 \pm 639$ vs. $1685 \pm 597 \mathrm{~mL}, \mathrm{P}=0.048)$, while there were no differences in the amount of allogeneic red blood cells needed, duration of $\mathrm{CPB}$, operation duration, and $\mathrm{CPB}$ cannulation (all $\mathrm{P}>0.05$ ). One patient in the $\mathrm{AKI}$ group required renal replacement therapy and died during the perioperative period.

\section{Risk factors for postoperative AKI}

Table 2 shows that age $(P=0.012)$, intraoperative urine volume $(P=0.048)$ were associated with the occurrence of postoperative AKI. Multivariable analysis indicated that age $(\mathrm{OR}=1.10,95 \% \mathrm{Cl}: 1.02-1.20$, $P=0.020)$ was independently associated with AKI occurrence.

\section{Discussion}

The results suggest that the patients undergoing unilateral radical nephrectomy and CPB-assisted inferior vena cava thrombectomy have a high AKI rate. Age is associated with the occurrence of AKI in such patients.

Renal cancer complicated with cancer-associated thrombosis in the inferior vena cava or even the atrium is challenging ${ }^{7-10}$. In the early days, targeted therapy was used in most cases. In recent years, some large surgical centers in China have been performing radical nephrectomy and inferior vena cava thrombectomy with the emergence of more advanced surgical instruments, anesthetic, and surgical techniques ${ }^{10,14-17}$. Compared with other therapies, radical nephrectomy combined with thrombectomy can significantly extend patients' overall survival and improve their 5 -year survival rate ${ }^{18}$. Cancerassociated embolism is graded according to the five-grade rating method of the Mayo Clinic, and the surgery is performed according to the grade. Grade 0 cancer-associated thrombosis can be removed without blocking the inferior vena cava. Grades I and II cancer-associated thrombosis can be removed by only blocking the proximal and distal inferior vena cava and the contralateral renal vein momentarily. For grades III and IV cancer-associated thrombosis, it is suggested to perform CPB to control the 
hemodynamics and cause deep hypothermic circulatory arrest (DHCA). Cancer-associated thrombosis in the right atrium should be completely removed, requiring $\mathrm{CPB}$ and $\mathrm{DHCA}{ }^{19}$. About $38 \%$ of the patients undergoing surgery have postoperative complications, and $4 \%-10 \%$ die during the perioperative period 20 . According to the literature, postoperative complications are mainly related to the grade of cancerassociated thrombosis. Specifically, the incidence of postoperative complications in patients with grade I cancer-associated thrombosis is $18 \%$ and increases to $20 \%$ in grade II, $26 \%$ in grade III, and $47 \%$ in grade IV ${ }^{21}$. Bleeding, pulmonary embolism, incision infection, and acute renal failure are common postoperative complications in patients with grade IV cancer-associated thrombosis, as reported in the literature ${ }^{7-10,22}$.

The occurrence of postoperative AKI might be related to the surgical procedures, such as the use of CPB, great blood pressure fluctuations due to poor control of intraoperative blood pressure, and use of colloids during surgery, so after the CPB-aided surgery for renal cancer complicated with cancer-associated thrombosis, in which one kidney is removed, the nephron number drops by half, and the patient is more prone to AKI. However, our results indicated that there was no obvious significant difference in CPB duration between patients with AKI or not.

For anesthesia during radical nephrectomy and CPB-aided inferior vena cava thrombectomy, the main difficulties are the risks of intraoperative bleeding and embolism due to detachment of the cancerassociated thrombosis when it is being removed during surgery, as well as instability of the circulatory system after vessel blocking, which would cause severe fluctuations and ischemic injury of vital organs $7-10,23$. Before surgery, the surgeon should have full access to the tumor's location and should grade the cancer-associated thrombosis and determine the specific surgical procedures. As the right renal vein is shorter than the left renal vein, renal cancer complicated with cancer-associated thrombosis in the inferior vena cava on the right is more common than on the left, and surgery for cancer-associated thrombosis of grade $\geq I I$ on the left is more difficult than that on the right. Moreover, sufficient amounts of blood products should be prepared before surgery because blood loss during the surgery for grade III cancerassociated thrombosis can be up to $2000 \mathrm{~mL}$, and the blood loss during surgery for grade IV cancerassociated thrombosis can be up to $4000 \mathrm{~mL}$. For patients needing CPB, plasma, fibrinogen, and platelets should also be prepared ${ }^{24}$. DHCA should be provided during the CPB-assisted surgery for grade IV cancer-associated thrombosis, but CPB and hypothermia can have a higher risk of acute renal failure ${ }^{25}$. The patient should be given appropriate volume enlargement before CPB and be given vasoactive drugs to maintain the central venous pressure at the higher normal value, to avoid a sharp drop in the volume of returned blood and the blood pressure after blocking. During blocking, the fluid infusion should be limited, and a-receptor agonists and venous dilators should be given to maintain the CVP at the lower normal value, to avoid acute heart failure or pulmonary edema due to a great volume of returned blood when the inferior vena cava is opened ${ }^{15}$. In this study, the difference in anesthesia duration between the two groups was not statistically significant, suggesting that anesthesia duration was not significantly related to postoperative renal function impairment or renal failure. Moreover, our research also demonstrated that surgical trauma in patients may have no obvious difference in non-AKI group and AKI group. Patients 
with renal cancer are older, have decreased physical functions and comorbidities, and have poorer glomerular filtration than young people, which causes a higher incidence of $A K{ }^{17}$. Results of this study corroborated such a conclusion since age was the only associated risk factor. Our results suggest that more attention should be paid to older patients after surgery.

This study has limitations. It was a retrospective study with a small sample size from a single center. This study carries all the biases inherent to retrospective analyses.

In conclusion, patients undergoing unilateral radical nephrectomy and CPB-assisted thrombectomy in the inferior vena cava or atrial pulmonary artery are at risk of postoperative AKI. Of such patients, older ones are at a higher risk of postoperative AKI.

\section{Methods}

The study protocol was approved by the Ethics Committees of the Jilin Cancer Hospital. All methods were performed in accordance with the relevant guidelines and regulations. The included patients all provided signed informed consent.

\section{Study design}

This retrospective study included patients who underwent unilateral radical nephrectomy and CPBassisted thrombectomy in the inferior vena cava and/or atrial pulmonary artery due to RCC, under general anesthesia, from December 2011 to June 2015, at the Department of Urology of Peking University First Hospital.

\section{Surgical intervention}

The patients received preoperative imaging examinations, including 4D cardiac imaging, pulmonary arteriovenous angiography, and peripheral angiography. They were examined in detail before anesthesia for liver and kidney functions and blood routine and electrolyte indicators, especially heart and lung functions. Anesthesia induction was performed with endotracheal intubation and $0.2-0.3 \mathrm{mg} / \mathrm{kg}$ of midazolam, $0.2-0.3 \mu \mathrm{g} / \mathrm{kg}$ of sufentanil, $0.6 \mathrm{mg} / \mathrm{kg}$ of rocuronium bromide, $1.5-2 \mathrm{mg} / \mathrm{kg}$ of propofol, and 2-3 ng/ $\mathrm{mL}$ of the remifentanil target control. Continuous infusion of $4-6 \mathrm{mg} / \mathrm{kg} / \mathrm{h}$ of propofol and intermittent infusion of sufentanil, midazolam, pancuronium bromide, or rocuronium bromide were performed for anesthesia maintenance. Internal jugular vein catheterization was performed for blood and fluid transfusion, and artery puncture was performed to monitor the patients' arterial pressure.

During unilateral radical nephrectomy, the patient was in the supine position. A median sternotomy was performed, and CPB was carried out through aortic cannulation or aortofemoral/right subclavian arterial cannulation based on the extent of tumor invasion. During CPB, the patient was given $3 \mathrm{mg} / \mathrm{kg}$ of heparin via a central venous catheter. The dose was adjusted to maintain the activated clotting time (ACT) between 480 and $600 \mathrm{~s}$. After CPB, the patient was given protamine antagonists. The patient's vital signs, 
ECG, central venous pressure (CVP), mean arterial pressure (MAP), oxyhemoglobin saturation, nasopharyngeal temperature, and rectal temperature were monitored during surgery.

\section{Observational indicators}

The demographic information, complications, and preoperative baseline serum creatinine (Scr) of patients were collected. The patient's intraoperative allogeneic red blood cells (U), CPB duration, operation duration, anesthesia duration, intraoperative urinary volume and stay time in ICU, the number of patients needing aortic cannulation during surgery, the number of patients who died during the perioperative period, and the number of patients needing the renal replacement therapy were observed and taken as assessment indicators.

\section{Diagnostic standard for AKI and grouping}

The Kidney Disease Improving Global Outcomes (KDIGO) criteria were used to diagnose postoperative $\mathrm{AKI}^{13}$. A patient was diagnosed with postoperative AKI if, after surgery, Scr increased by $0.3 \mathrm{mg} / \mathrm{dL}(26.5$ $\mu \mathrm{mol} / \mathrm{L}$ ) or more in $48 \mathrm{~h}$ or the Scr increased to 1.5 times the baseline value or higher in 7 days, or the urine volume was $<0.5 \mathrm{ml} / \mathrm{kg} / \mathrm{h}$ for 6 consecutive $\mathrm{h}$. The patients were grouped into the AKI group and the non-AKI group.

\section{Statistical analysis}

SPSS 15.0 (SPSS Inc., Chicago, IL, USA) was used for data analysis. Continuous variables are expressed as means \pm standard deviations (SD) and were analyzed using Student's t-test. Categorical variables are presented as $n(\%)$ and were analyzed using the chi-square test. Univariable and multivariable logistic regression analysis was used to identify the risk factors for AKI. Variables with $\mathrm{P}<0.10$ in the univariable analyses were included in the multivariable analysis. Two-sided P-values $<0.05$ were deemed statistically significant.

\section{Declarations}

\section{ACKNOWLEDGMENTS}

None.

\section{FUNDING}

None.

\section{COMPETING INTERESTS}

All authors declare that they have no competing interests.

\section{References}


1. Capitanio, U. \& Montorsi, F. Renal cancer. Lancet. 387, 894-906 (2016).

2. Ferlay, J., et al. Cancer incidence and mortality patterns in Europe: Estimates for 40 countries and 25 major cancers in 2018. Eur J Cancer. 103, 356-387 (2018).

3. Bray, F., et al. Global cancer statistics 2018: GLOBOCAN estimates of incidence and mortality worldwide for 36 cancers in 185 countries. CA Cancer J Clin. 68, 394-424 (2018).

4. NCCN Clinical Practice Guidelines in oncology (NCCN Guidelines). Kidney Cancer. Version 2.2021. National Comprehensive Cancer Network, Fort Washington, 2020.

5. Escudier, B., et al. Renal cell carcinoma: ESMO Clinical Practice Guidelines for diagnosis, treatment and follow-up. Ann Oncol. 27, v58-v68 (2016).

6. Siegel, R. L., Miller, K. D., Fuchs, H. E. \& Jemal, A. Cancer Statistics, 2021. CA Cancer J Clin. 71, 7-33 (2021).

7. Oltean, M. A., et al. Renal Cell Carcinoma With Extensive Tumor Thrombus Into the Inferior Vena Cava and Right Atrium in a 70-Year-Old Man. Am J Mens Health. 13, 1557988319846404 (2019).

8. Psutka, S. P. \& Leibovich, B. C. Management of inferior vena cava tumor thrombus in locally advanced renal cell carcinoma. Ther Adv Urol. 7, 216-229 (2015).

9. Quencer, K. B., Friedman, T., Sheth, R. \& Oklu, R. Tumor thrombus: incidence, imaging, prognosis and treatment. Cardiovasc Diagn Ther. 7, S165-S177 (2017).

10. Qi, J., et al. Management of renal cell carcinoma with tumor thrombus in renal vein and the inferior vena cava. Ann Vasc Surg. 24, 1089-1093 (2010).

11. Tanaka, M., et al. Prognostic factors of renal cell carcinoma with extension into inferior vena cava. Int J Urol. 15, 394-398 (2008).

12. Blute, M. L., Leibovich, B. C., Lohse, C. M., Cheville, J. C. \& Zincke, H. The Mayo Clinic experience with surgical management, complications and outcome for patients with renal cell carcinoma and venous tumour thrombus. BJU Int. 94, 33-41 (2004).

13. Khwaja, A. KDIGO clinical practice guidelines for acute kidney injury. Nephron Clin Pract. 120, c179184 (2012).

14. Karnes, R. J. \& Blute, M. L. Surgery insight: management of renal cell carcinoma with associated inferior vena cava thrombus. Nat Clin Pract Urol. 5, 329-339 (2008).

15. Cai, S. L., Luo, J. D. \& Wang, Q. [Diagnosis and treatment of renal cancer complicated with cancerassociated thrombosis in the inferior vena cava]. Chin J Urol. 26, 516-519 (2005).

16. Liu, Z., Ma, L. L. \& Tian, X. J. [Clinical experience in treating Mayo grade 0 to grade IV inferior vena cava cancer-associated thrombosis with radical nephrectomy and inferior vena cava thrombectomy]. Chin J Urol. 38, 842-847 (2017).

17. Ma, L. L. \& Liu, Z. [Findings in diagnosis of renal cancer complicated with cancer-associated thrombosis in the inferior vena cava in hepatic and upper segments]. Chin J Urol. 38, 481-484 (2017).

18. Shuch, B., et al. Intraoperative thrombus embolization during nephrectomy and tumor thrombectomy: critical analysis of the University of California-Los Angeles experience. J Urol. 181, 492-498; 
discussion 498-499 (2009).

19. Sahmeddini, M. A., et al. The Effect of Intraoperative Restricted Normal Saline during Orthotopic Liver Transplantation on Amount of Administered Sodium Bicarbonate. Iran J Med Sci. 39, 247-253 (2014).

20. Ishikawa, S., Griesdale, D. E. \& Lohser, J. Acute kidney injury after lung resection surgery: incidence and perioperative risk factors. Anesth Analg. 114, 1256-1262 (2012).

21. Bihorac, A., et al. Long-term risk of mortality and acute kidney injury during hospitalization after major surgery. Ann Surg. 249, 851-858 (2009).

22. Kheterpal, S., et al. Development and validation of an acute kidney injury risk index for patients undergoing general surgery: results from a national data set. Anesthesiology. 110, 505-515 (2009).

23. Shaw, A., Swaminathan, M. \& Stafford-Smith, M. Cardiac surgery-associated acute kidney injury: putting together the pieces of the puzzle. Nephron Physiol. 109, p55-60 (2008).

24. Roh, G. U., et al. Incidence and risk factors of acute kidney injury after thoracic aortic surgery for acute dissection. Ann Thorac Surg. 94, 766-771 (2012).

25. Martinez-Salamanca, J. I., et al. Prognostic impact of the 2009 UICC/AJCC TNM staging system for renal cell carcinoma with venous extension. Eur Urol. 59, 120-127 (2011).

\section{Tables}

Table 1. Characteristics of the patients 


\begin{tabular}{|llll|}
\hline Characteristics & Non-AKI $(\mathrm{n}=16)$ & AKI $(\mathrm{n}=15)$ & $\mathrm{P}$ \\
\hline Male, $\mathrm{n}(\%)$ & $8(50.0)$ & $9(60.0)$ & 0.552 \\
\hline Age (mean \pm SD, years) & $48.5 \pm 12.9$ & $59.0 \pm 8.7$ & 0.012 \\
\hline Body mass index (mean $\left.\pm \mathrm{SD}, \mathrm{kg} / \mathrm{m}^{2}\right)$ & $22.5 \pm 2.3$ & $23.4 \pm 3.2$ & 0.407 \\
\hline Hypertension, $\mathrm{n}(\%)$ & $4(25.0)$ & $8(53.3)$ & 0.183 \\
\hline Smoking, $\mathrm{n}(\%)$ & $6(37.5)$ & $5(33.3)$ & 0.611 \\
\hline Diabetes, $\mathrm{n}(\%)$ & $3(18.8)$ & $4(26.7)$ & 0.793 \\
\hline Preoperative Scr (mean $\pm \mathrm{SD}, \mu \mathrm{mol} / \mathrm{L})$ & $101.0 \pm 28.2$ & $85.4 \pm 20.9$ & 0.094 \\
\hline Preoperative CCr (mean $\pm \mathrm{SD}, \mathrm{mL} / \mathrm{min})$ & $73.1 \pm 19.9$ & $76.3 \pm 25.8$ & 0.705 \\
\hline Intraoperative allogeneic red blood cell (mean $\pm \mathrm{SD}, \mathrm{U})$ & $5.47 \pm 2.88$ & $5.31 \pm 2.96$ & 0.884 \\
\hline CPB duration (mean $\pm \mathrm{SD}, \mathrm{min})$ & $32.5 \pm 17.1$ & $41.4 \pm 24.3$ & 0.247 \\
\hline Operation duration (mean $\pm \mathrm{SD}, \mathrm{min})$ & $456 \pm 93$ & $474 \pm 94$ & 0.604 \\
\hline Anesthesia duration (mean $\pm \mathrm{SD}, \mathrm{min})$ & $495 \pm 95$ & $536 \pm 99$ & 0.249 \\
\hline Intraoperative urine volume (mean $\pm \mathrm{SD}, \mathrm{mL})$ & $1685 \pm 597$ & $1225 \pm 639$ & 0.048 \\
\hline Aortic cannulation, $\mathrm{n}(\%)$ & $9(56.3)$ & $12(80.0)$ & 0.458 \\
\hline Stay time in ICU (mean \pm SD, $\mathrm{h})$ & $51.7 \pm 25.1$ & $90.0 \pm 80.3$ & 0.088 \\
\hline Perioperative death, $\mathrm{n}(\%)$ & 0 & $1(6.7)$ & \\
\hline Renal replacement therapy, $\mathrm{n}(\%)$ & 0 & $1(6.7)$ & \\
\hline
\end{tabular}

AKI: acute kidney injury; Scr: serum creatinine; CCr: creatinine clearance; CPB: cardiopulmonary bypass; ICU: intensive care unit.

Table 2. Logistic regression analysis of risk factors for AKI

\begin{tabular}{|llll|}
\hline Characteristics & Univariable & Multivariable & \\
\cline { 2 - 4 } & $\mathrm{P}$ & $\mathrm{OR}(95 \% \mathrm{Cl})$ & $\mathrm{P}$ \\
\hline Age & 0.012 & $1.10(1.02-1.20)$ & 0.020 \\
\hline Preoperative Scr & 0.094 & $0.97(0.93-1.00)$ & 0.082 \\
\hline Intraoperative urine volume & 0.048 & - & - \\
\hline Stay time in ICU & 0.088 & - & - \\
\hline
\end{tabular}


AKI: acute kidney injury; OR: odds ratio; Cl: confidence interval; Scr: serum creatinine; ICU: intensive care unit. 